BULL. AUSTRAL. MATH. SOC.

$62 \mathrm{HI7}, 62 \mathrm{G} 10,62 \mathrm{G} 20,62 \mathrm{E} 20$

VOL. $28(1983), 295-297$.

\title{
ON CHOOSING A GOODNESS-OF-FIT TEST
}

\section{TIMOTHY R.C. READ}

There has been much discussion regarding the respective merits of the Pearson $x^{2}$ and the log-likelihood ratio tests of goodness-of-fit under the multinomial distribution.

In this thesis it is shown that both these test statistics, and a variety of others which are less well known, belong to a larger family of goodness-of-fit statistics. This fact raises the questions:

(1) Which properties do the members of this family have in common and how do they differ?

(2) How can we decide which statistic is the "best" choice for any given conditions?

Under well known regularity conditions both Pearson's $x^{2}$ and the log-likelihood ratio statistics are asymptotically equivalent and follow an asymptotic $x^{2}$ distribution. This result is extended to the general family. Furthermore asymptotic properties of the family are derived under a less well known, but intuitively sensible, set of regularity conditions, of which the most important is that the number of classes tends to infinity with the sample size. In this case the statistics follow an asymptotic normal distribution, but the asymptotic equivalence no longer exists.

Various criteria for assessing the relative merits of these test

Received 29 August 1983. Thesis submitted to Flinders University of South Australia, May 1982. Degree approved June 1983. Supervisors: Dr N.A. Cressie, Professor J.N. Darroch, Dr M. Jirina.

Copyright Clearance Centre, Inc. Serial-fee code: 0004-9727/83 $\$ A 2.00+0.00$. 
statistics are considered both asymptotically and in finite samples. In the asymptotic case it is shown under the first regularity scenario that the Pitman asymptotic relative efficiency is 1 for any two members of the family. However using the criterion of exact Bahadur efficiency the loglikelihood ratio statistic is optimal. Under the second regularity scenario, the Pitman asymptotic relative efficiency indicates that Pearson's $x^{2}$ statistic is optimal provided some extra conditions (including symmetry of the null hypothesis) are satisfied.

In the finite sample case, the error incurred in applying the asymptotic distributional results is assessed through second order asymptotics and small sample exact studies. Closer approximations to the first three moments of the family, under the asymptotic $\chi^{2}$ regularity conditions, indicate that there exist members of the family whose moments converge to the limiting $\chi^{2}$ moments faster than those of either Pearson's $x^{2}$ or the log-likelihood ratio statistic. Furthermore the asymptotic equivalence of the family under these conditions, is shown to be only a first order effect.

Exact and approximate critical regions are compared under the symmetric hypothesis for specific members of the family using the $x^{2}$ and normal approximations discussed above, together with two corrected $\chi^{2}$ approximations. It is shown that there are statistics in the family preferable to the log-likelihood ratio statistic, in that their true test levels are more closely approximated by the asymptotic results. Furthermore, exact power comparisons under alternatives with large single cell perturbations show that the power of the well known tests can always be improved by choosing other statistics from the family.

Minimum distance parameter estimation is discussed with respect to the general family of statistics. Conditions are given under which estimators based on different family members are optimal.

Other goodness-of-fit statistics have been proposed in the literature which do not belong to the general family. An asymptotic assessment of some of these alternative statistics indicates no particular superiority over the family of statistics discussed here. 
Finally a brief analogy is drawn with various results in the literature of generalized information measures. 